\title{
The small heat shock proteins from Acidithiobacillus ferrooxidans: gene expression, phylogenetic analysis, and structural modeling
}

\author{
Daniela A Ribeiro', Luiz EV Del Bem', Renato Vicentini ${ }^{1}$, Lúcio FC Ferraz ${ }^{1}$, Mario T Murakami ${ }^{2}$ and \\ Laura MM Ottoboni ${ }^{*}$
}

\begin{abstract}
Background: Acidithiobacillus ferrooxidans is an acidophilic, chemolithoautotrophic bacterium that has been successfully used in metal bioleaching. In this study, an analysis of the A. ferrooxidans ATCC 23270 genome revealed the presence of three SHSP genes, Afe_1009, Afe_1437 and Afe_2172, that encode proteins from the HSP20 family, a class of intracellular multimers that is especially important in extremophile microorganisms.

Results: The expression of the sHSP genes was investigated in A. ferrooxidans cells submitted to a heat shock at $40^{\circ} \mathrm{C}$ for 15, 30 and 60 minutes. After 60 minutes, the gene on locus Afe_1437 was about 20-fold more highly expressed than the gene on locus Afe_2172. Bioinformatic and phylogenetic analyses showed that the sHSPs from A. ferrooxidans are possible non-paralogous proteins, and are regulated by the $\sigma^{32}$ factor, a common transcription factor of heat shock proteins. Structural studies using homology molecular modeling indicated that the proteins encoded by Afe_1009 and Afe_1437 have a conserved $\alpha$-crystallin domain and share similar structural features with the sHSP from Methanococcus jannaschii, suggesting that their biological assembly involves 24 molecules and resembles a hollow spherical shell.
\end{abstract}

Conclusion: We conclude that the sHSPs encoded by the Afe_1437 and Afe_1009 genes are more likely to act as molecular chaperones in the A. ferrooxidans heat shock response. In addition, the three SHSPs from A. ferrooxidans are not recent paralogs, and the Afe_1437 and Afe_1009 genes could be inherited horizontally by A. ferrooxidans.

\section{Background}

Acidithiobacillus ferrooxidans is an acidophilic, chemolithoautotrophic bacterium that derives energy from the oxidation of ferrous iron, elemental sulfur and reduced sulfur compounds [1]. This bacterium has been successfully used in bioleaching to recover metals from lowgrade sulfide ores. During the bioleaching process, $A$. ferrooxidans is subjected to extreme growth conditions, such as temperature increase, $\mathrm{pH}$ fluctuations, nutrient starvation, and the presence of heavy metals [2], all of which can affect the efficiency of metal recovery.

Temperature change is one of the most common environmental stresses that can influence essential

\footnotetext{
* Correspondence: ottoboni@unicamp.br

${ }^{1}$ Center for Molecular Biology and Genetic Engineering (CBMEG), State University of Campinas - UNICAMP, Candido Rondon Avenue 400, 13083-875 - Campinas, SP, Brazil

Full list of author information is available at the end of the article
}

bacterial processes such as energy transduction and growth. All organisms tend to respond to environmental stresses with a rapid transient increase in heat shock protein (HSP) synthesis. HSPs act either as molecular chaperones, mediating the correct folding and assembly of proteins, or as proteases, irreversibly degrading unfolded proteins [3]. The HSPs are usually classified according to their molecular weights, and the small HSPs (denoted sHSPs) include the categories HSP100, HSP90, HSP70, HSP60, and HSP20.

The sHSPs are characterized by a molecular mass of between 12 and $43 \mathrm{kDa}$ and the presence of 80 to 100 residues that constitute the $\alpha$-crystallin domain, which is flanked by $\mathrm{C}$ - and $\mathrm{N}$-terminals that present lower similarity. The $\mathrm{N}$-terminus is critical to $\alpha$-HSP activity in vivo, playing a role in $\alpha$-HSP oligomerization and substrate binding $[4,5]$. The $\alpha$-crystallin domain is known to possess a molecular chaperone role [6], and 
the C-terminal extension maintains $\alpha$-HSP solubility, stability, and chaperone activity [4].

The sHSPs have been extensively studied due to their importance in protecting cellular proteins and maintaining cellular viability under intensive stress conditions, which is particularly important for extremophile microorganisms. Interestingly, most extremophiles posses one or two sHSPs, and species harboring at least 3 sHSP genes are mostly from the Archea domain. However, three sHSP genes have been identified in the genome of A. ferrooxidans ATCC 23270 [7].

Xiao et al. [8] showed that there could be significant differences in the expression levels of $A$. ferrooxidans ATCC 23270 sHSP genes in response to heat shock. These findings suggest that $A$. ferrooxidans sHSP genes may be controlled by different regulatory mechanisms, which could be related to specialized functions of the genes. In this study, the expression levels of three sHSP genes (Afe_1009, Afe_1437, and Afe_2172) were investigated in the $A$. ferrooxidans LR strain subjected to heat shock. Phylogenetic analysis and comparative molecular modeling were used to provide new insights concerning the structure and function of the sHSPs from $A$. ferrooxidans.

\section{Methods}

\section{Bacterial strain and growth conditions}

The Brazilian strain $A$. ferrooxidans LR [9] was grown at $30^{\circ} \mathrm{C}$ and $250 \mathrm{rpm}$ in modified T\&K liquid medium [10] containing $0.4 \mathrm{~g} / \mathrm{L} \mathrm{K}_{2} \mathrm{HPO}_{4} .3 \mathrm{H}_{2} \mathrm{O}, 0.4 \mathrm{~g} / \mathrm{L} \mathrm{MgSO}_{4} .7 \mathrm{H}_{2} \mathrm{O}$, $0.4 \mathrm{~g} / \mathrm{L}\left(\mathrm{NH}_{4}\right)_{2} \mathrm{SO}_{4}$, and $33.4 \mathrm{~g} / \mathrm{L} \mathrm{FeSO} 4.7 \mathrm{H}_{2} \mathrm{O}$. The $\mathrm{pH}$ was adjusted to 1.8 with sulfuric acid. For the heat shock experiments, $A$. ferrooxidans LR cells were grown in $\mathrm{T} \& \mathrm{~K}$ liquid medium until $50 \%$ oxidation of $\mathrm{Fe}^{2+}$ was reached. The cells were then collected, inoculated into $100 \mathrm{ml}$ of $\mathrm{T} \& \mathrm{~K}$ liquid medium, and incubated at $40^{\circ} \mathrm{C}$ and $250 \mathrm{rpm}$ for 15,30 and 60 minutes.

\section{RNA isolation}

The total RNA was isolated from three independent $A$. ferrooxidans cultures, according to the procedure described by Paulino et al. [11]. The cells were suspended in a solution containing $1 \mathrm{mM}$ EDTA, $100 \mathrm{mM}$ $\mathrm{LiCl}$, and $100 \mathrm{mM}$ Tris- $\mathrm{HCl}$, at $\mathrm{pH}$ 7.5. The RNA fraction was extracted with phenol/chloroform/isoamyl alcohol $(25: 24: 1, \mathrm{v} / \mathrm{v} / \mathrm{v})$ containing $10 \%(\mathrm{w} / \mathrm{v})$ SDS, precipitated at $-20^{\circ} \mathrm{C}$ with $2 \%(\mathrm{w} / \mathrm{v})$ potassium acetate at $\mathrm{pH} 5.5$ and $100 \%(\mathrm{v} / \mathrm{v})$ ethanol, and resuspended in DEPC-treated water. The RNA was treated with DNase (Invitrogen) for $1 \mathrm{~h}$ at $37^{\circ} \mathrm{C}$, and stored at $-70^{\circ} \mathrm{C}$.

\section{Quantitative real-time PCR (qRT-PCR)}

The relative expressions of Afe_1009, Afe_1437, and Afe_2172 were determined by qRT-PCR [12]. The
cDNAs were synthesized with the ThermoScript RTPCR system kit (Invitrogen). The alaS gene was used as the endogenous control [13]. The primers used in the experiments were designed with the Primer3 program http://frodo.wi.mit.edu/, employing the entire coding region of the selected genes from the $A$. ferrooxidans ATCC 23270 genome (Table 1). The specificity of the primers was confirmed by PCR using genomic DNA from $A$. ferrooxidans LR.

The qRT-PCR experiments were performed in triplicate using a 7500 Real Time PCR System (Applied Biosystems), and threshold cycle $(\mathrm{Ct})$ numbers were determined using Real Time System RQ Study Software v. 1.3.1 (Applied Biosystems). The qRT-PCR reactions were performed in triplicate using Platinum SYBR Green qPCR SuperMix-UDG (Invitrogen). After thermal cycling, a dissociation (melting) curve analysis was performed to ensure the specificity of the amplifications and the absence of primer-dimer and unspecific amplifications. The relative gene expression was calculated according to the comparative critical threshold method $(\Delta \Delta \mathrm{TC})$ described by Livak and Schmittgen [14]. The statistical significance of the qRT-PCR data was determined using the Student's t-test ( $\mathrm{p}$-value $\leq 0.05$ ).

\section{Bioinformatics analysis}

The A. ferrooxidans ATCC 23270 genome (J. Craig Venter Institute - http://cmr.jcvi.org/cgi-bin/CMR/Genome) was used to search for genes encoding sHSPs. CLUSTAL W was employed to align the sHSP sequences from $A$. ferrooxidans with sequences found in other bacteria. The alignment was edited with the GeneDoc program [15].

Prediction of the transcription start site was performed with BPROM software (Softberry, Inc.). A widely accepted theoretical informational approach was adopted to identify potential $\sigma^{32}$ sites $[16,17]$. Since the $\sigma^{32}$ binding site comprises two conserved blocks (-35 and -10$)$, separated by a gap of variable length, two positional weight matrices (PWM) were generated, each one based on complementary information from the -35 and -10 binding sites. The frequency matrix was based on a set of eighteen $V$. cholerae $\sigma^{32}$ promoters [18], including the extended $\sigma^{32}$ promoter, with 6 positions in the -35 element and 8 positions in the -10 element, separated by a spacer of variable length. Using the PWMs as a scoring function, putative -35 and -10 regions of $\sigma^{32}$ were searched on 200 bases upstream from the ATG start codon of the A. ferrooxidans sHSP genes. Each site was scored for its degree of matching to the $\sigma^{32}-35$ and -10 PWMs.

\section{Phylogenetic analysis}

A search was performed against all complete bacterial genomes (1295 genomes on 08/03/2010), using NCBI's 
Table 1 Primers used in the real-time PCR experiments.

\begin{tabular}{cllc}
\hline Target gene & Forward primer $\left(\mathbf{5}^{\prime} \boldsymbol{\rightarrow} \mathbf{3} \mathbf{3}^{\prime}\right)$ & Reverse primer $\left(\mathbf{5}^{\prime} \boldsymbol{\rightarrow} \rightarrow \mathbf{3}^{\prime}\right)$ & Amplicon length $(\mathbf{b p})$ \\
\hline Afe_1009 & CCGAAATACCTGAGGTCAA & TCCCTTCTCCTCCTTCTCC & 91 \\
Afe_1437 & GTATTGAAGGCGGAGATGC & TCTTCTCCTTGACGCCACT & 118 \\
Afe_2172 & AGGTAATCTTCAGCGGCAAC & TAGGGGATCTCCAGACGATG & 97 \\
\hline
\end{tabular}

microbial genome BLAST tool http://www.ncbi.nlm.nih. gov/sutils/genom_table.cgi?organism $=$ microb and the protein sequences from Afe_1009, Afe_1437 and Afe_2172 as queries. The 20 best hits for each A. ferrooxidans sHSP were selected to build an alignment using MAFFT v6.717b http://align.bmr.kyushu-u.ac.jp/ mafft/software/. The alignment containing 76 aligned residues was used to produce a maximum likelihood (ML) tree using PhyML 3.0 software http://atgc.lirmm. fr/phyml/. The PAM matrix procedure [19] was used to calculate genetic distances, and statistical support for the nodes employed aLRT statistics [20].

\section{Molecular modeling}

PSI-BLAST search against the Protein Data Bank (PDB) using the three A. ferrooxidans sHSPs (Afe_1009, Afe_1437, and Afe_2172) resulted only in templates with low sequence identity $(<28 \%)$. However, fold assignment searches using the pGenTHREADER algorithm implemented in the PSIPRED server [21] returned two structures that had significant scores, both of which displayed well-conserved $\alpha$-crystallin domains. The crystal structures of HSP16.9 from wheat (wHSP16.9, PDB entry code: 1GME) [22] and HSP16.5 from Methanococcus jannaschii (MjHSP16.5, PDB entry code: 1SHS) were used as threedimensional templates for molecular modeling of the $\alpha$ crystallin domain. The $\mathrm{N}$-terminal region was modeled using only the wHSP16.9 structure as template. Template and target sequences were aligned using the mGenThreader server [23], and were carefully examined to confirm the alignment accuracy. Comparative protein modeling by satisfaction of spatial restraints was carried out using the program MODELLER 9v7 [24]. Fifty models were built for each sHSP from $A$. ferrooxidans, and all models were evaluated with the DOPE potential. Models of each protein with the lower global score were selected for explicit solvent molecular dynamics (MD) simulation, using GROMACS [25] to check for stability and consistency. The overall and local quality of the final model was assessed by VERIFY3D [26], PROSA [27] and VADAR [28]. Threedimensional structures were displayed, analyzed, and compared using the programs COOT [29] and PyMoL [30].

\section{Results and Discussion}

The sHSPs from $A$. ferrooxidans

Search of the A. ferrooxidans ATCC 23270 genome (J. Craig Venter Institute) revealed the presence of three
sHSP genes (Afe_1009, Afe_1437, and Afe_2172) belonging to the HSP20 family. According to Han and co-workers [31], about $71 \%$ of the microbial organisms with completed annotated genomes possess one or two sHSP genes, and $10 \%$ of the Archaea species have more than three sHSP-related genes. Notably, the genome of Bradyrhizobium japonicum (a rhizobial species) possesses 13 sHSP-related genes [32].

Laksanalamai and Robb [7] showed that the degree of identity of the sHSPs from several extremophiles possessing only one sHSP was $75 \%$, while the identity of sHSPs from the same organism ranged from 20 to $50 \%$. The low sequence identity for the $A$. ferrooxidans sHSPs (Table 2) is therefore intriguing.

Afe_1009, Afe_1437, and Afe_2172 are not organized in an operon in the A. ferrooxidans genome. Indeed, most of the known sHSP genes are not arranged in operons [33,34], with some exceptions such as the Escherichia coli ibpAB operon, which contains two sHSP genes (ibpA and $i b p B$ ) [35,36], and Bradyrhizobium japonicum, which has sHSP genes found as independent units and others grouped in the same operon [32].

\section{sHSP genes expression in A. ferrooxidans LR cells subjected to heat shock}

qRT-PCR was used to determine the transcript levels of the Afe_1009, Afe_1437, and Afe_2172 genes in A. ferrooxidans LR cells grown at $30^{\circ} \mathrm{C}$ (control) or subjected to a $40^{\circ} \mathrm{C}$ heat shock for 15,30 and 60 minutes (Figure $1)$. The qRT-PCR results indicate that after 60 minutes all three sHSP genes were significantly up-regulated ( $\mathrm{p}<$ 0.05 and fold change $\geq 2.0$ ), although the expression level of Afe_2172 was considerably lower than the expression levels of Afe_1437 and Afe_1009. The expression level for Afe_1437 was 20-fold higher than that observed for Afe_2172, and 11.5-fold higher than the expression level of Afe_1009. Xiao et al. [8] observed a similar pattern of expression for the Afe_1437 gene. Our results for Afe_1009 and Afe_2172 were dissimilar to those obtained by Xiao et al. [8]. However, this comparison may not be reliable due to differences in the $A$. ferrooxidans strains as well as the heat shock experiments used in the two studies.

The observed differences in the expressions of the three $A$. ferrooxidans sHSP genes suggest possible regulatory differences. In many bacteria, the $\sigma^{32}$ factor regulates the expression of the sHSP-encoding genes in a 
Table 2 Physical and chemical parameters of the three sHSPs from A. ferrooxidans.

\begin{tabular}{lllllll}
\hline Gene & Length & $\begin{array}{l}\text { Molecular weight } \\
\text { (Da) }\end{array}$ & $\begin{array}{l}\text { Theoretical } \\
\text { pl }\end{array}$ & $\begin{array}{l}\text { Identity/similarity to } \\
\text { Afe_1009 }\end{array}$ & $\begin{array}{l}\text { Identity/similarity to } \\
\text { Afe_1437 }\end{array}$ & $\begin{array}{l}\text { Identity/similarity to } \\
\text { Afe_2172 }\end{array}$ \\
\hline Afe_1009 & 145 & 16934 & 6.20 & - & $29 / 58 \%$ & $26 / 47 \%$ \\
Afe_1437 & 148 & 16680 & 5.43 & $29 / 58 \%$ & - & $22 / 53 \%$ \\
Afe_2172 & 134 & 16401 & 5.60 & $26 / 47 \%$ & $22 / 53 \%$ & - \\
\hline
\end{tabular}

temperature-dependent manner [35]. Under stress conditions, the transcription of heat shock genes is induced following a rapid and transient increase of this factor [37]. A bioinformatics analysis was therefore performed in the deduced -10 and -35 regions of the three sHSP genes. The results indicated that the three genes had possible $\sigma^{32}$-dependent promoters (Figure 2). In the work undertaken by Xiao et al. [8], $\sigma^{32}$-dependent promoters were only found for the Afe_1437 and Afe_2172 genes. However, the disparities between the two studies can be explained by the different in silico strategies chosen.

In $A$. ferrooxidans, the -35 motif at the $\sigma^{32}$ binding site appears to be more conserved than the -10 motif. The same occurs for the $V$. cholerae and the E. coli $\sigma^{32}$ consensus sequences [18]. In spite of the different expression levels observed for the A. ferrooxidans sHSP genes, the bioinformatics analyses did not reveal any other type of regulation mechanism (data not shown). However, within the $\sigma^{32}$-regulated genes, alternative mechanisms of regulation are possible. Münchbach and co-workers [32] used subtractive two-dimensional gel electrophoresis to identify a set of 10 sHSPs in B. japonicum subjected to a temperature shift from $28^{\circ} \mathrm{C}$ to $43^{\circ}$ C. These authors observed that the amounts of the
sHSPs were quite dissimilar, suggesting the existence of a diverse regulatory repertoire.

Phylogenetic analysis and comparative sequence analysis The ML analysis suggested that the three sHSPs from $A$. ferrooxidans are not recent paralogs (Figure 3 ). This finding is in accordance with the low sequence similarity between the sHSPs from $A$. ferrooxidans (Table 2 and Figure 3). The sequence divergence among the $A$. ferrooxidans sHSPs is likely to be the consequence of horizontal transfer of one or even two genes; however, the possibility of divergent evolution [38] caused by different selective pressures cannot be fully discarded. To gain more insight into the origins of the A. ferrooxidans sHSPs, the CG content of each gene was compared with the average $C G$ content of $A$. ferrooxidans coding-genes ( $~ 59 \%$ of CG). The CG contents of Afe_1437 (46.53\%) and Afe_1009 (47.71\%) were statistically different from the average $A$. ferrooxidans CG content $\left(\mathrm{p}<0.01 ; \mathrm{x}^{2}=\right.$ 11.7766 and $x^{2}=9.4510$, respectively), while for Afe_2172 (58.76\%) there was no significant difference $\left(x^{2}=0.1025\right)$. These findings suggest that Afe_1437 and Afe_1009 could be inherited horizontally by A. ferrooxidans. Interestingly, the closely related species $A$. caldus from the same genus has only one sHSP gene, which is

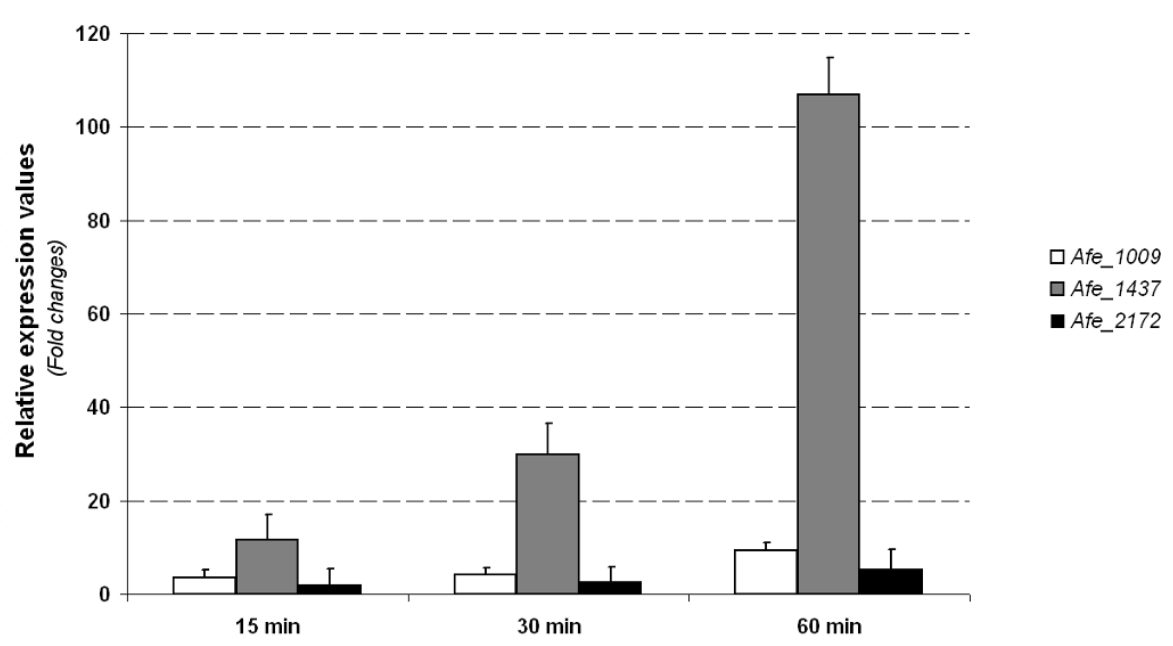

Figure 1 Expression of the shsp genes from A. ferrooxidans LR. Expression of the genes located at loci Afe_1009, Afe_1437, and Afe_2172 in A. ferrooxidans $L R$ cells submitted to heat shock $\left(40^{\circ} \mathrm{C}\right)$ at different times $(15,30$, and $60 \mathrm{~min})$. The expression values, obtained by Real time PCR, are relative to the ones obtained from cells maintained at $30^{\circ} \mathrm{C}$. 


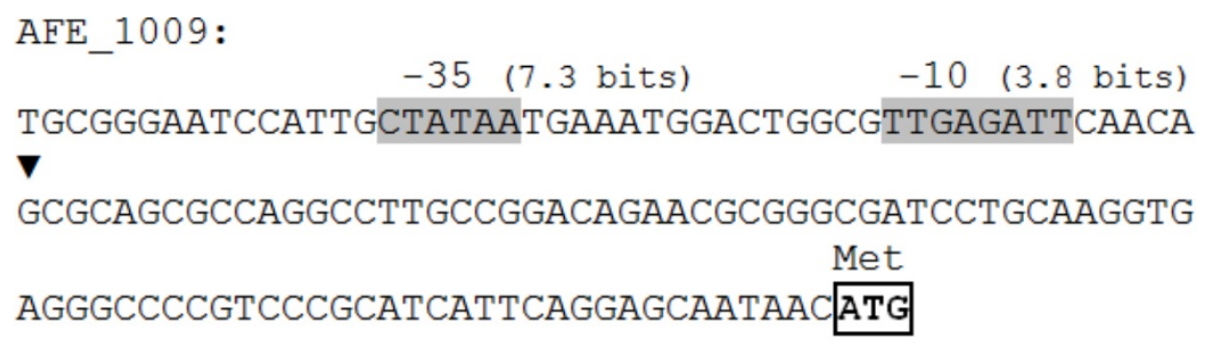

AFE_1437:

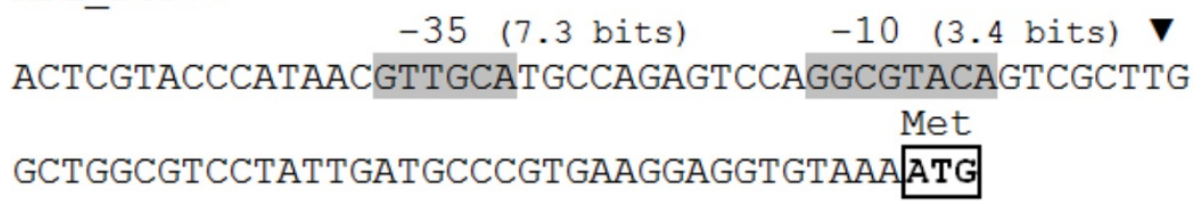

AFE_2172: -35 (5.4 bits) $\quad-10$ (4.5 bits) CCCGGCGTCAGCCTGGTTGTCGCCGCCAGGCTGGAGAGGTATAGCCGCA $\boldsymbol{\nabla}$ AАAАTACGCTACAATATTGGGACGTCATGGACGCCGATCAGGGGGAGTG Met GGGGATG

Figure 2 Nucleotide sequences of the $5^{\prime}$-upstream regions of the three genes, Afe_1009, Afe_1437, and Afe_2172, which encode sHSPs in $\boldsymbol{A}$. ferrooxidans. Transcription start sites predicted by the BPROM program and promoter sequences recognized by the $\boldsymbol{\sigma}^{32}$ factor are indicated by black triangles and by shadowed-bold letters, respectively. The first codon of the coding sequence is indicated by boxed letters. The total information content of the $\sigma^{32}$ boxes $(-35$ and -10$)$ is shown in bits.

the possible ortholog of A. ferrooxidans Afe_1437. Considering the hypothesis of horizontal transfer origins of Afe_1437 and Afe_1009, it is likely that A. caldus has lost the ortholog of Afe_2172 (putative original sHSP) and maintained the ortholog of Afe_1437. In this scenario, the lateral transference that originated Afe_1437 occurred prior to the divergence between these two species.

Figure 4 shows the alignment of the amino acid sequences of the three sHSPs from A. ferrooxidans with other sHSP sequences, including sequences from the gamma-proteobacteria subdivision. As shown in Figure 4, the sHSPs from A. ferrooxidans harbor the well-conserved $\alpha$-crystallin domain and all elements considered essential for their oligomerization, and therefore for their chaperone activity. However, the Afe_2172 protein has a very short $\mathrm{C}$-terminus that is rarely observed in sHSPs from other bacteria. The only other exception is a sHSP from Bordetella avium, a bacterium that causes an upper respiratory tract disease in avian species (Figure 4). This feature can either decrease their ability to oligomerize or modulate their chaperone activity.
Moreover, the C-terminal region of sHSPs from some bacteria presents highly conserved cysteine residues. These residues have been proposed to enable the sHSPs to sense changes under oxidizing conditions of the environment, and to translate these changes into differences in protein conformation and chaperone activity [39]. Also, in some plant species, a conserved methionine-rich sequence at the $\mathrm{N}$-terminal region has been proposed to offer a redox control of chaperone-like activity and dynamics of the oligomeric structure [40]. However, these conserved cysteine residues at the C-terminus, as well as the conserved methionine-rich motif at the N-terminus, were not found in the sHSPs phylogenetically related to A. ferrooxidans (Figure 4), which suggests an absence of such control in the sHSPs belonging to the gamma-proteobacteria subdivision.

The N-terminal region showed no significant sequence similarity to other sHSPs with well-defined chaperone activity (groups $\mathrm{C}$ and $\mathrm{D}$ ), but secondary structure prediction tools indicated that all of the sequences analyzed had the propensity to form the $\alpha$-helical structures that are considered key elements for substrate binding and 


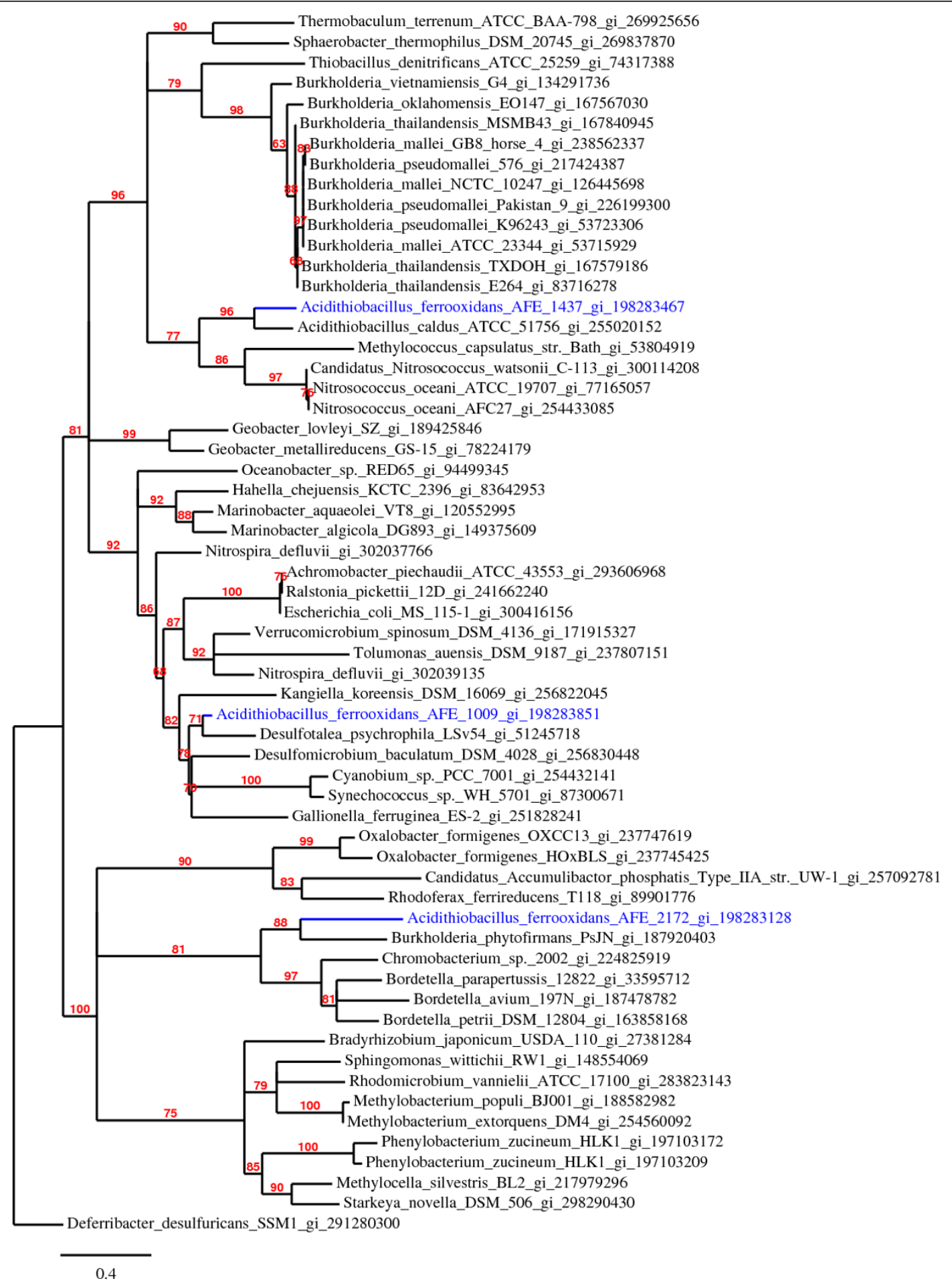

Figure 3 Inferred phylogenetic relationships among the $\boldsymbol{A}$. ferrooxidans and closely related bacterial sHSPs. The 20 closest related bacterial protein sequences to each A. ferrooxidans sHSP were retrieved by a BLAST search against 1295 completed bacteria genomes (see Methods section). The topology was obtained by ML using 76 aligned amino acids residues. Distances were calculated by PAM matrix and the statistical confidence of the nodes was calculated by aLRT test. Branches with aLRT values lower than $50 \%$ were collapsed. GeneBank accession numbers are shown in front of the species name. 


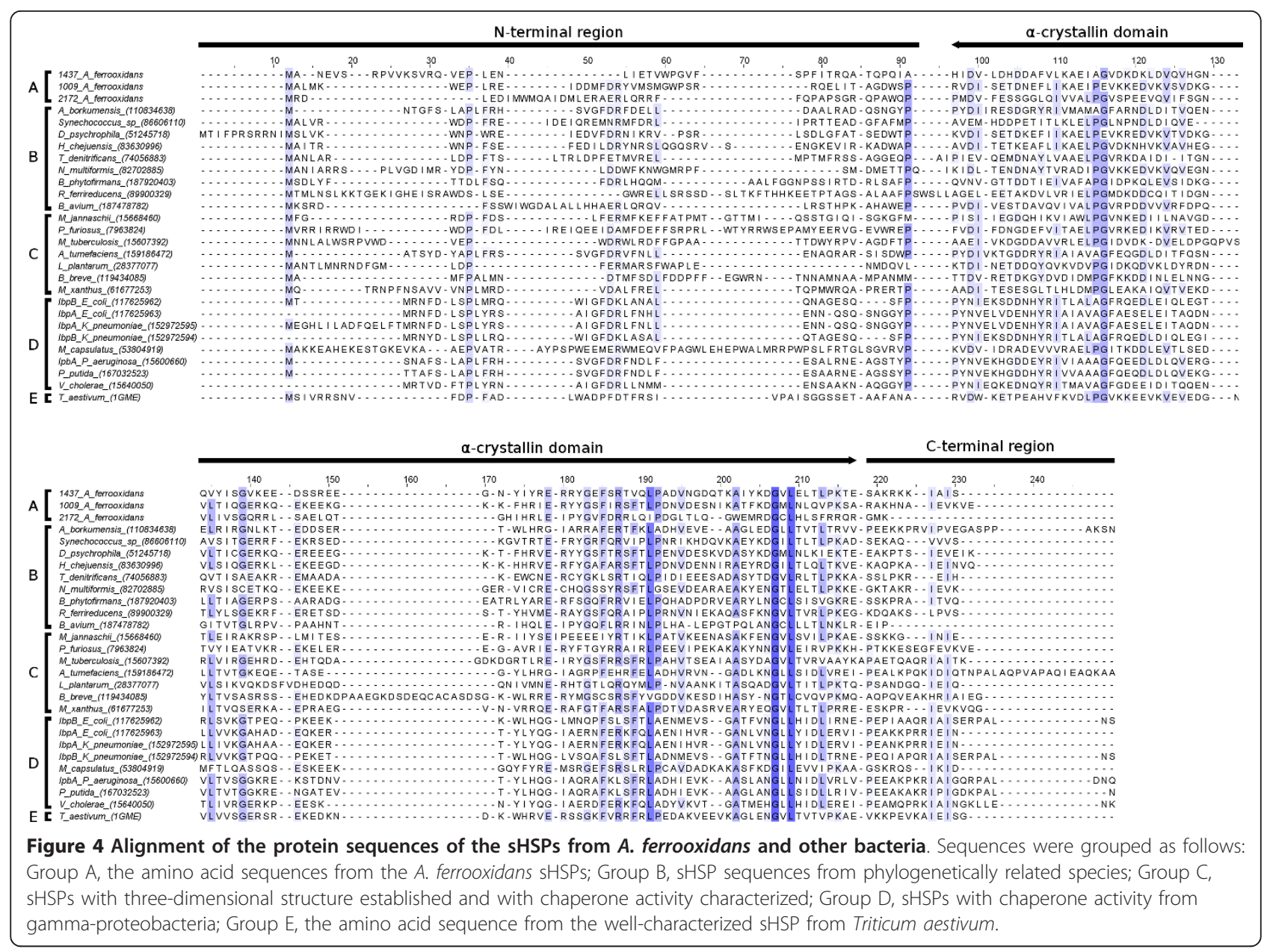

stabilization of the oligomeric structure. Furthermore, the $\mathrm{N}$-terminal region alone was capable of interacting with denatured proteins [41], and its truncation reduces the chaperone activity of sHSPs [42]. These findings emphasize that this region contains the substrate binding site, and is therefore important for the chaperone activity.

\section{Structural modeling of the sHSPs from A. ferrooxidans}

In silico three-dimensional models of the proteins encoded by Afe_1009, Afe_1437, and Afe_2172 displayed excellent global and local stereochemical properties, with a Z-score (PROSA server) of around -3.5 and all residues lying within the allowed regions of the Ramachandran plot. A good Z-score means that it is within the range of scores typically found for native proteins of similar size. RMSD analysis of the template crystal structures and the developed models resulted in values below $0.5 \AA$ for the main-chain backbone of the $\alpha$-crystallin domain, suggesting that the models were suitable for structural and comparative analyses.
The $\alpha$-crystallin domains of the proteins encoded by Afe_1009, Afe_1437, and Afe_2172 share similar structural features with other sHSPs from both prokaryotic and eukaryotic organisms. This domain (residues 46$135)$ shows a $\beta$-sandwich fold composed of seven $\beta$ strands in two sheets (Figure 5). The N-terminal region (residues 1-45), encompassing two helical segments, was only observed in the structure of wHSP16.9 from wheat [22]. In the wHSP16.9 structure, the N-terminal helices participate in the stabilization of the oligomeric structure, establishing interactions with the adjacent $\alpha$-crystallin domain [22]. The C-terminal extension (136-148) displays a random coil conformation and has a critical role in the formation of the oligomeric state. However, different to the proteins encoded by Afe_1437 and Afe_1009, the Afe_2172 protein has a rare shortened Cterminus, which may prevent the formation of a stable oligomer and could be involved in the modulation of the protein chaperone activity. Canonically, the long loop, which is responsible for dimerization, is fully conserved, and the identification of functional regions by surface-mapping of phylogenetic information, using the 

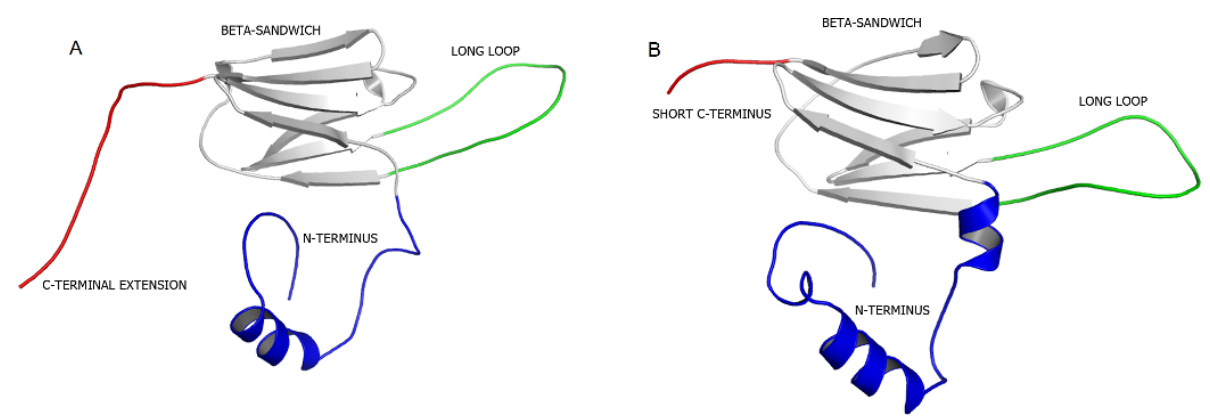

Figure $\mathbf{5}$ Cartoon representation of the modeled structure of the sHSPs from A. ferrooxidans. (A) Proteins encoded by loci Afe_1009 and Afe_1437. (B) Protein encoded by loci Afe_2172. The b-sandwich domain, long loop, and N- and C-terminal regions are colored in light grey, green, dark blue, and red, respectively.

ConSurf web server [43], indicates that all residues considered essential for dimerization are fully conserved in the three sHSPs from A. ferrooxidans.

In order to gain insights into the oligomeric state of the proteins encoded by Afe_1437 and Afe_1009, which possess the extended C-terminus, analysis was performed of the structural determinants required for assembling into either a dodecameric double disk (wHSP16.9) or a spherical shell composed of 24 monomers (MjHSP16.5). In both the wHSP16.9 and the MjHSP16.5 structures, the intermolecular interactions made by the C-terminal extension are virtually identical, despite the fact that the C-terminus of wHSP16.9 requires two different orientations to form the oligomer. This ability of the C-terminus to adopt two conformations resides in the amino acid segment between the strands $\beta 9$ and $\beta 10$, which permits a hinge movement. Analysis of the C-terminus contacts in the MjHSP16.5 structure showed that the segment between the strands $\beta 9$ and $\beta 10$ adopts a conformation stabilized by hydro-

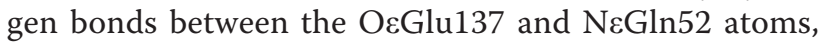
and the carbonyl oxygen of the Glu137 and N $\zeta$ Lys142 atoms. Surprisingly, these contacts are not found in the wHSP16.9 structure, due to the presence of a second Pro residue at position 142 that enables the segment to fold into a stable motif, generating a 6-residue segment (KAEVKK) with high flexibility, which allows the hinge movement. In both Afe_1437 and Afe_1009 protein sequences, this segment does not contain a proline residue at the same relative position, and the residues populating this segment have all the requirements to form a stable motif in the same way as the MjHSP16.5 structure. Thus, based on our structural findings, we suggest that both Afe_1437 and Afe_1009 proteins behave like the prokaryotic sHSP from $M$. jannaschii, adopting a 24-molecule hollow spherical shell. However, additional experimental data obtained using techniques that can provide insights into hydrodynamic behavior, such as dynamic light scattering, ultra-centrifugation, size-exclusion chromatography and small angle X-ray scattering, are required to confirm our in silico predictions.

\section{Conclusions}

In this study, we have demonstrated that the expression level of the A. ferrooxidans Afe_1437 gene is considerable higher than that of the Afe_2172 gene, and that the three sHSP genes harbor possible $\sigma^{32}$-dependent promoters. The three sHSPs from A. ferrooxidans are not recent paralogs, while the genes Afe_1437 and Afe_1009 can be inherited horizontally by $A$. ferrooxidans. This suggests that the sHSPs encoded by Afe_1437 and Afe_1009 are more likely to act as molecular chaperones in the A. ferrooxidans heat shock response. These findings were corroborated by molecular modeling showing that both Afe_1437 and Afe_1009 proteins behave like the prokaryotic sHSP from $M$. jannaschii, a well characterized sHSP with chaperone activity.

\section{Acknowledgements}

This work was supported by grant 02/07642-3 from Fundação de Amparo à Pesquisa do Estado de São Paulo (FAPESP). DAR had a fellowship from Coordenação de Aperfeiçoamento de Pessoal de Nível Superior (CAPES). LMMO received a research fellowship from Conselho Nacional de Desenvolvimento Científico e Tecnológico (CNPq).

\section{Author details}

${ }^{1}$ Center for Molecular Biology and Genetic Engineering (CBMEG), State University of Campinas - UNICAMP, Candido Rondon Avenue 400, 13083-875 - Campinas, SP, Brazil. ${ }^{2}$ National Biosciences Laboratory (LNBio), National Laboratory of Energy and Materials Research (CNPEM), Giuseppe Máximo Scolfaro Street 10000, 13083-970 - Campinas, SP, Brazil.

\section{Authors' contributions}

All authors have read and approved the final manuscript. DAR and LMMO conceived the idea and designed the experiments. DAR and LFCF executed the RTq-PCR experiments. DAR wrote the manuscript. RV performed the bioinformatics analysis; LEVDB, the phylogenetic analysis; and MTM, the molecular modeling.

Received: 21 July 2011 Accepted: 7 December 2011 Published: 7 December 2011 
References

1. Kelly DP, Wood AP: Reclassification of some species of Thiobacillus to the newly designated genera Acidithiobacillus gen. nov., Halothiobacillus gen. nov. and Thermithiobacillus gen. nov. Int J Syst Evol Microbiol 2000, 50:511-516.

2. Rawlings DE: Characteristics and adaptability of iron and sulfur-oxidizing microorganisms used for the recovery of metals from minerals and their concentrates. Microb Cell Fac 2005, 4(13):1-15.

3. Lindquist S: The heat-shock response. Ann Rev Biochem 1986, 55:1151-1191.

4. Sun Y, MacRae TH: Small heat shock proteins: molecular structure and chaperone function. Cell Mol Life Sci 2005, 62:2460-2476.

5. Giese KC, Basha E, Catague BY, Vierling E: Evidence for an essential function of the $\mathrm{N}$ terminus of a small heat shock protein in vivo, independent of in vitro chaperone activity. Proc Natl Acad Sci USA 2005 102(52):18896-18901.

6. Horwitz J: Alpha-crystallin can function as molecular chaperone. Proc Nat Acad Sci USA 1992, 89:10449-10453.

7. Laksanalamai P, Robb FT: Small heat shock proteins from extremophiles: a review. Extremophiles 2004, 8(1):1-11.

8. Xiao S, Chao J, Wang W, Fang F, Qui G, Liu X: Real-time RTq-PCR analysis of the heat-shock response of Acidithiobacillus ferrooxidans ATCC 23270. Folia Biol (Praha) 2009, 55(1):1-6.

9. Garcia O Jr, da Silva LL: Differences in growth and iron oxidation among Thiobacillus ferrooxidans cultures in the presence of some toxic metals. Biotechnol Lett 1991, 13:567-570.

10. Tuovinen OH, Kelly DP: Biology of Thiobacillus ferrooxidans in relation to the microbiological leaching of sulphide ore. Zeitschrift fur Allgemeine Mikrobiologie 1972, 12:311-346.

11. Paulino LC, Mello MP, Ottoboni LMM: Differential gene expression in response to copper in Acidithiobacillus ferrooxidans analyzed by RNA arbitrarily primed polymerase chain reaction. Electrophoresis 2002, 23:520-527.

12. Carlos C, Reis FC, Vicentini R, Madureira DJ, Ottoboni LMM: The rus operon genes are differentially regulated when Acidithiobacillus ferrooxidans LR is kept in contact with metal sulfides. Curr Microbiol 2008, 57:375-380.

13. Yarzábal A, Appia-Ayme C, Ratouchniak J, Bonnefoy V: Regulation of the expression of the Acidithiobacillus ferrooxidans rus operon encoding two cytochromes c, a cytochrome oxidase and rusticyanin. Microbiol 2004, 150:2113-2123

14. Livak KJ, Schmittgen TD: Analysis of relative gene expression data using real-time quantitative RTq-PCR and the 2(-Delta Delta $C(T)$ ) method. Methods 2001, 25:402-408.

15. Chenna R, Sugawara H, Koike T, Lopez R, Gibson TJ, Higgins DJ, Thompson JD: Multiple sequence alignment with the Clustal series programs. Nucleic Acids Res 2003, 31:3497-3500.

16. Schneider TD: Information content of individual genetic sequences. $J$ Theor Biol 1997, 189:427-441.

17. Reents $H$, Münch $R$, Dammeyer $T$, Jahn D, Härtig E: The Fnr regulon of Bacillus subtilis. J Bacteriol 2006, 188:1103-1112.

18. Slamti L, Livny J, Waldor MK: Global gene expression and phenotypic analysis of a Vibrio cholerae rpoH deletion mutant. J Bacteriol 2007. 189(2):351-362.

19. Dayhoff $\mathrm{MO}$, Schwartz RC, Orcutt BC: A model of evolutionary change in proteins. In Atlas of protein sequence and structure. Volume 5. Silver Spring 1978:(3):301-310.

20. Anisimova M, Gascuel O: Approximate likelihood ratio test for branches: A fast, accurate and powerful alternative. Syst Biol 2006, 55:539-552.

21. McGuffin $L$, Bryson $K$, Jones DT: The PSIPRED protein structure prediction server. Bioinformatics 2000, 16(4):404-405.

22. van Montfort RL, Basha E, Friedrich KL, Slingsby C, Vierling E: Crystal structure and assembly of a eukaryotic small heat shock protein. Nat Struct Mol Biol 2001, 8(12):1025-30.

23. McGuffin LJ, Jones DT: Improvement of the GenTHREADER method for genomic fold recognition. Bioinformatics 2003, 19(7):874-881.

24. Fiser A, Sali A: Modeller: generation and refinement of homology-based protein structure models. Methods Enzymol 2003, 374:461-491.

25. Lindahl E, Hess B, van der Spoel D: GROMACS 3.0: a package for molecular simulation and trajectory analysis. J Mol Model 2001, 7:306-317.

26. Eisenberg D, Lüthy $R$, Bowie JU: VERIFY3D: Assessment of protein models with three-dimensional profiles. Methods Enzymol 1997, 277:396-404.
27. Wiederstein $M$, Sippl MJ: ProSA-web: interactive web service for the recognition of errors in three-dimensional structures of proteins. Nucleic Acids Res 2007, 35:W407-W410.

28. Willard L, Ranjan A, Zhang H, Monzavi H, Boyko RF, Sykes BD, Wishar DS: VADAR: a web server for quantitative evaluation of protein structure quality. Nucleic Acids Res 2003, 31(13):3316-3319.

29. Emsley P, Cowtan K: Coot: model-building tools for molecular graphics. Acta Crystallogr D Biol Crystallogr 2004, 60:2126-2132.

30. DeLano WL: The PyMOL Molecular Graphics System. DeLano Scientific San Carlos, CA, USA; 2002.

31. Han MJ, Yun H, Lee SY: Microbial small heat shock proteins and their use in biotechnology. Biotechnol Adv 2008, 26:591-609.

32. Münchbach $M$, Nocker $A$, Narberhaus F: Multiple small heat shock proteins in Rhizobia. J Bacteriol 1999, 181(1):83-90.

33. Roy SK, Hiyama T, Nakamoto H: Purification and characterization of the 16-kDa heat-shock-responsive protein from the thermophilic cyanobacterium Synechococcus vulcanus, which is an alpha-crystallinrelated, small heat shock protein. Eur J Biochem 1999, 262(2):406-416.

34. Tomoyasu T, Takaya A, Sasaki T, Nagase T, Kikuno R, Morioka M, Yamamoto T: A new heat shock gene, AgsA, which encodes a small chaperone involved in suppressing protein aggregation in Salmonella enterica serovar typhimurium. J Bacterio/ 2003, 185(21):6331-6339.

35. Allen SP, Polazzi JO, Gierse JK, Easton AM: Two novel heat shock genes encoding proteins produced in response to heterologous protein expression in Escherichia coli. J Bacteriol 1992, 174(21):6938-6947.

36. Kuczynska-Wisnik D, Laskowska E, Taylor A: Transcription of the ibpB heatshock gene is under control of $\sigma^{32}$ and $\sigma^{34}$ promoters, a third regulon of heat-shock response. Biochem Biophys Res Commun 2001, 284:57-64.

37. Gao H, Wang Y, Liu X, Yan T, Wu L, Alm E, Arkin A, Thompson DK, Zhou J: Global transcriptome analysis of the heat shock response of Shewanella oneidensis. J Bacterio/ 2004, 186(22):7796-7803.

38. Ingram VM: Gene evolution and the haemoglobins. Nature 1961, 4(189):704-708.

39. Graf PCF, Jakob U: Redox-regulated molecular chaperones. Cell Mol Life Sci 2002, 59:1624-1631.

40. Gustavsson N, Kokke BP, Anzeilius AB, Boelens WC, Sundby C: Substitution of conserved methionines by leucines in chloroplast small heat shock protein results in loss of redox-response but retained chaperone-like activity. Protein Sci 2001, 10:1785-1793.

41. Fu X, Zhang $H$, Zhang $X$, Cao $Y$, Jião W, Liu C, Song $Y$, Abulimiti A, Chang Z: A dual role for the $\mathrm{N}$-terminal region of Mycobacterium tuberculosis Hsp 16.3 in self-oligomerization and binding denaturing substrate proteins. J Biol Chem 2005, 280:6337-6384.

42. Usui K, Hatipoglu OF, Ishii N, Yohda M: Role of the N-terminal region of the crenarchaeal sHSP, Sthsp14.0, in thermal-induced disassembly of the complex and molecular chaperone activity. Biochem Biophys Res Commun 2004, 315:113-118.

43. Goldenberg O, Erez E, Nimrod G, Ben-Tal N: The ConSurf-DB: precalculated evolutionary conservation profiles of protein structures. Nucleic Acids Res 2009, 37:D323-D327.

doi:10.1186/1471-2180-11-259

Cite this article as: Ribeiro et al:: The small heat shock proteins from Acidithiobacillus ferrooxidans: gene expression, phylogenetic analysis, and structural modeling. BMC Microbiology 2011 11:259.

\section{Submit your next manuscript to BioMed Central and take full advantage of:}

- Convenient online submission

- Thorough peer review

- No space constraints or color figure charges

- Immediate publication on acceptance

- Inclusion in PubMed, CAS, Scopus and Google Scholar

- Research which is freely available for redistribution 